\title{
Comparação da experiência de cárie em moradores de dois municípios brasileiros com e sem a fluoretação das águas de abastecimento público
}

\author{
Comparison of caries experience in residents of two Brazilian \\ municipalities with and without fluoridation of the public water supplies
}

\section{La comparación de la experiencia de caries en los residentes en dos municipios brasileños con y sin la fluoración del abastecimiento público de agua}

Maria Paula Maciel Rando-Meirelles | mpaula_rando@yahoo.com.br

Universidade Estadual de Campinas, Faculdade de Odontologia de Piracicaba. Piracicaba, Brasil.

Fabricio Narciso Olivati | fabriciolivati@hotmail.com

Universidade Estadual de Campinas, Faculdade de Odontologia de Piracicaba. Piracicaba, Brasil.

Décio Henrique Franco | deciohenriquefranco@yahoo.com.br

Universidade Estadual de Campinas, Faculdade de Odontologia de Piracicaba. Piracicaba, Brasil.

Telmo Oliveira Bittar $\mid$ telmobittar@hotmail.com

Universidade Estadual de Campinas, Faculdade de Odontologia de Piracicaba. Piracicaba, Brasil.

Tais Cristina Nascimento Marques | tais.odontologia@hotmail.com

Universidade Estadual de Campinas, Faculdade de Odontologia de Piracicaba. Piracicaba, Brasil.

Maria da Luz Rosario de Sousa | luzsousa@fop.unicamp.br

Universidade Estadual de Campinas, Faculdade de Odontologia de Piracicaba. Piracicaba, Brasil.

\section{Resumo}

A fluoretação da água de abastecimento público é uma medida eficaz e de baixo custo para a prevenção da cárie dentária. Este artigo se baseia em um estudo que comparou a experiência de cárie entre dois municípios do Brasil, São Paulo e Manaus, na época, com e sem fluoretação de suas águas. O estudo utilizou dados do projeto SBBrasil 2010 que avaliou três índices para 2.176 indivíduos: ceo-d (dentição infantil com cárie, extraídos e obturados), CPO-D (quantidade de dentes cariados, perdidos e obturados em adultos) e SiC (Significant Caries Index, uma variação do CPO-D). Os resultados mostraram que os índices foram maiores em Manaus quando comparados com os de São Paulo, para indivíduos com 5 e 12 anos de idade e pertencentes à faixa de 15 a 19 anos. O município de São Paulo apresentou melhor condição de saúde bucal em crianças e adolescentes, e não houve diferença entre os índices para adultos e idosos, demonstrando que parte da população com acesso a água fluoretada foi beneficiada pelo método.

Palavras-chave: cárie dentária; fluoretação; epidemiologia; políticas públicas de saúde; saúde coletiva; avaliação de pesquisa em saúde. 


\begin{abstract}
The public water supply fluoridation is an effective measure and is cost-effective in preventing tooth decay. This article bases on a study that compared the caries experience between two municipalities in Brazil, São Paulo and Manaus, at the time, with and without fluoridation of its water. The study used the projeto SBBrasil 2010 (SBBrazil project 2010) data that evaluated three indices for 2.176 individuals: $\mathrm{dmft}$ (decay, missing, filled teeth for primary dentition), DMFT (number of decayed, missing and filled in adults) and SiC (Significant Caries Index, a variation of DMTF). The results showed that the rates were higher in Manaus when compared to São Paulo, for 5 and 12 years old children and for young people who are 15 to 19 years old. The city of São Paulo showed better bucal health status in children and adolescents, and it was not found difference in indices for adults and the elderly, demonstrating that part of the population with access to fluoridated water was benefited by the method.
\end{abstract}

Keywords: caries; fluoridation; epidemiology; public health policy; public health; health research evaluation.

\title{
Resumen
}

La fluoración del agua potable pública es una medida de bajo costo y eficaz en la prevención de la caries. Este artículo es basado en estudio que comparó las experiencias de caries entre dos municipios de Brasil, Sao Paulo y Manaos, en la ocasión, con y sin la fluoración de su agua. El estudio utilizó los datos del projeto SBBrasil 2010 (proyecto SBBrasil2010) que evaluó tres índices para 2.176 individuos: ceo-d (dientes de los niños con caries, extraídos y obturados), CPO-D (número de dientes cariados, perdidos y obturados en adultos) y SiC (Significant Caries Index, una variación de CPO-D). Los resultados mostraron que las tasas eran más altas en Manaos, en comparación con Sao Paulo, para individuos de 5 y 12 años de edad y para jóvenes entre 15 y 19 años. La ciudad de Sao Paulo mostró un mejor estado de salud bucal en niños y adolescentes, y no evidenció diferencias entre los adultos y los ancianos, lo que demuestra que parte de la población con acceso a agua fluorada se vio beneficiada por el método.

Palabras clave: caries; fluoruración; epidemiología; políticas públicas de salud; salud pública; evaluación de investigación en salud.

Contribuição dos autores:

Os autores do artigo tiveram igual participação na concepção e desenho do estudo, na aquisição e análise dos dados, na análise estatística, na preparação e redação do manuscrito e na revisão crítica final do conteúdo intelectual.

Declaração de conflito de interesses: Os autores declaram não haver conflito de interesse.

Fontes de financiamento: Não houve.

Considerações éticas: Por se tratar de estudo com dados secundários de domínio público e sem possibilidade de identificação dos sujeitos, dispensou-se a submissão do projeto à comitê de ética em pesquisa.

Agradecimento/Contribuições adicionais: Não houve.

Histórico do artigo: Submetido: 09.mar.2016 | Aceito: 13.out.2016 | Publicado: 23.dez.2016

Apresentação anterior: Não houve

Licença CC BY-NC atribuição não comercial. Com essa licença é permitido acessar, baixar (download), copiar, imprimir, compartilhar, reutilizar e distribuir os artigos, desde que para uso não comercial e com a citação da fonte, conferindo os devidos créditos de autoria e menção à Reciis. Nesses casos, nenhuma permissão é necessária por parte dos autores ou dos editores. 


\section{Introdução}

A fluoretação das águas de abastecimento público representa uma das principais estratégias populacionais de controle da cárie dentária, sendo recomendada pelas principais organizações de saúde do mundo ${ }^{1-2}$.

Atualmente, a fluoretação das águas de abastecimento está implantada em aproximadamente 50\% dos municípios brasileiros, beneficiando uma população com mais de 100 milhões de pessoas ${ }^{3}$. No entanto, a eficiência da fluoretação depende da sua continuidade e da regularidade de sua concentração ótima na água, que no Brasil varia entre 0,6 e 0,8 mg F/L, podendo ser aceitos, em algumas regiões de menor temperatura climática, valores até $0,9 \mathrm{mg} \mathrm{F} / \mathrm{L}^{3-5}$.

O benefício desse método na prevenção da doença cárie é extensivo a todas as faixas etárias da população coberta pelo sistema de tratamento de água, no qual o fluoreto é adicionado. O Centro de Controle e Prevenção de Doenças ${ }^{2}$, dos Estados Unidos, admite que o poder preventivo da água fluoretada é de $40 \%$ a $70 \%$ em crianças, reduzindo também a perda de dentes em adultos entre 40\% e 60\%.

Em revisões sistemáticas, os resultados demonstraram redução média de 30\% a 50\% na experiência de cárie dentária de indivíduos residentes em regiões com água fluoretada quando comparados com moradores de outras regiões desprovidas da medida ${ }^{6-8}$. Para Narvai ${ }^{9}$, essa medida apresenta um enorme potencial de "universalização" além de contribuir para que as pessoas tenham acesso à água tratada, talvez a mais importante ação de saúde pública. Por isso, pode-se considerar socialmente injusto não a realizar ou interrompê-la.

Entretanto, mesmo sendo a fluoretação da água de abastecimento um elemento essencial para a prevenção da cárie dentária ${ }^{10-11}$, no Brasil, importantes e populosas cidades não possuem a adição do fluoreto em seus sistemas de abastecimento público de água, privando o acesso de suas populações aos benefícios preventivos proporcionados pela medida.

Dessa forma, o objetivo deste estudo é comparar a experiência de cárie entre dois municípios de grande porte e que são capitais estaduais brasileiras, São Paulo e Manaus, respectivamente com e sem fluoretação nas águas dos seus sistemas de abastecimento público de água potável, conforme dados de 2010.

\section{Aspectos metodológicos}

O município de Manaus apresenta um IDH (Índice de Desenvolvimento Humano) médio de 0,774 e, de acordo com o Censo 2010, tem um total de 1.802.014 habitantes ${ }^{12}$. Desde sua origem até 2010 o município de Manaus não tinha ainda adicionado o íon fluoreto em suas águas de abastecimento público. A fluoretação da água em Manaus teve início após o ano de 2012. O município de São Paulo, maior cidade da América do Sul, também conforme o Censo 2010, possui um IDH médio de 0,841 e uma população de 11.244.369 de habitantes ${ }^{12}$.

O município de São Paulo já tem implantado o sistema de fluoretação de águas para abastecimento público desde 31 de outubro de 1985. Em 1991, aproximadamente 97\% da população residente na cidade tinha acesso à água tratada, clorada e fluoretada ${ }^{13}$.

Este estudo foi realizado com base nos resultados do Levantamento epidemiológico do Projeto SBBrasil 2010 - Pesquisa Nacional de Saúde Bucal14, conduzido pelo Ministério da Saúde nos anos de 2009 e 2010. No SBBrasil 2010 foi aplicada uma técnica de amostragem probabilística por conglomerados em três estágios, o que permitiu a produção de inferências para cada uma das macrorregiões brasileiras, por porte do município e para cada idade ou grupo etário.

As equipes de campo foram treinadas, em oficina com duração de 16 horas, com o objetivo de discutir a operacionalização das etapas do trabalho, as atribuições de cada participante e assegurar um grau 
aceitável de uniformidade nos procedimentos. A calibração dos examinadores envolveu pelo menos oito períodos de quatro horas de trabalho ${ }^{14}$.

Os exames odontológicos seguiram a metodologia proposta pela Organização Mundial da Saúde (OMS). Utilizaram-se espelho bucal plano e a sonda da OMS (sonda CPI), sob luz natural, com o examinador e a pessoa examinada sentados. Além da experiência de cárie avaliada pelo índice CPO-D, no momento do exame também foram verificadas as necessidades de tratamento odontológico, segundo os critérios propostos pela $\mathrm{OMS}^{14}$.

O índice CPO-D, originalmente formulado por Klein e Palmer em 1937, permanece sendo o mais utilizado em todo o mundo, mantendo-se como o ponto básico de referência para o diagnóstico das condições dentais e para a formulação e avaliação de programas de saúde bucal. A sigla CPO-D significa Dentes "Cariados, Perdidos e Obturados", ainda que a denominação mais correta, neste último caso, seja "Restaurado"; para efeitos do índice, se mantém a inicial “O” como uma concessão à sua melhor sonoridade.

Neste estudo foi utilizado o índice ceo-d que, escrito em letras minúsculas, corresponde ao CPO-D quando se trata da dentição decídua, ou seja, a dentição infantil, mas inclui só os dentes cariados (c), com extração indicada (e) e obturados (o), excluindo os extraídos devido às dificuldades para fazer uma separação entre os que o foram por causa de cárie e os perdidos pelo processo natural de esfoliação dentária.

O índice SiC (Significant Caries Index, uma variação do CPO-D) foi empregado para definir a gravidade da cárie no terço do grupo que apresentou maior experiência da doença. Para a análise dos resultados utilizou-se o teste de qui-quadrado e Mann-Whitney, com nível de significância de 5\%.

Foram examinados um total de 2.176 indivíduos, assim distribuídos: em Manaus (AM), 5 anos ( $n=204$ ), 12 anos ( $\mathrm{n}=148), 15$ a 19 anos ( $\mathrm{n}=146), 35$ a 44 anos (n=229), e 56 a 65 anos (n=181); e, em São Paulo (SP), 5 anos $(n=224), 12$ anos $(n=233), 15$ a 19 anos $(n=183), 35$ a 44 anos $(n=373)$, e 56 a 65 anos $(n=255)^{14}$.

O município de Manaus foi escolhido para esta comparação porque havia a certeza de que nunca havia tido suas águas de abastecimento fluoretadas até o ano de 2012 e, para este estudo, considerou-se se um município foi fluoretado, ou não, conforme o critério apresentado por Narvai colaboradores ${ }^{15}$, segundo o qual é considerada fluoretada a cidade em que a população esteve exposta ao benefício, continuamente, durante pelo menos cinco anos.

Em conformidade com a Resolução 196/96 do Conselho Nacional de Saúde (CNS), relativa às pesquisas em seres humanos, o Projeto SBBrasil 2010 foi submetido ao Comitê de Ética em Pesquisa do Ministério da Saúde, sendo aprovado e recebendo registro na Comissão Nacional de Ética em Pesquisa (Conep), do CNS, sob o número 15.498 em 7 de janeiro de 2010.

\section{Resultados}

O Gráfico 1 demonstra os índices CPO-D e SiC relativos aos analisados com 5 e 12 anos de idade e aos jovens pertencentes à faixa etária de 15 a 19 anos. Tanto os índices ceo-d / CPO-D quanto o SiC foram maiores no município de Manaus quando comparado a São Paulo, no caso dos que tinham 5 e 12 anos ( $<<0,001)$. E não apresentou diferença estatística significante para a faixa etária de 15 a 19 anos. 


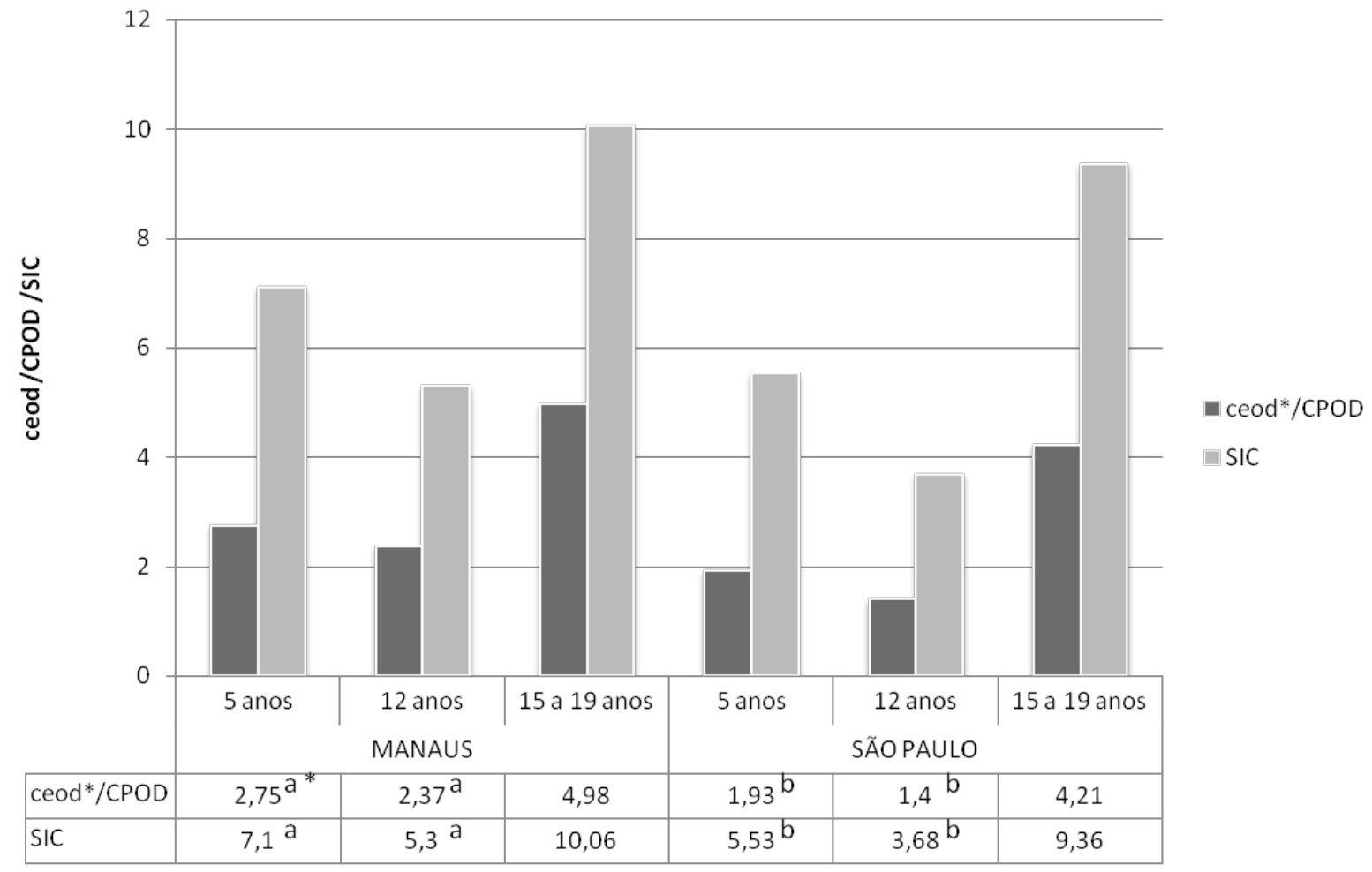

Gráfico 1 - Índice ceo-d (5 anos), CPO-D (12 e 15 anos) e SiC de Manaus e São Paulo, 2010 $\mathrm{a}, \mathrm{b}$ Letras distintas na linha indicam diferença estatisticamente significante dentro de cada grupo etário. Fonte: Elaborado pelos autores, 2015.

O Gráfico 2 demonstra a comparação dos mesmos índices na população adulta e idosa. Não houve diferença significativa para o índice CPOD nem para o SiC.

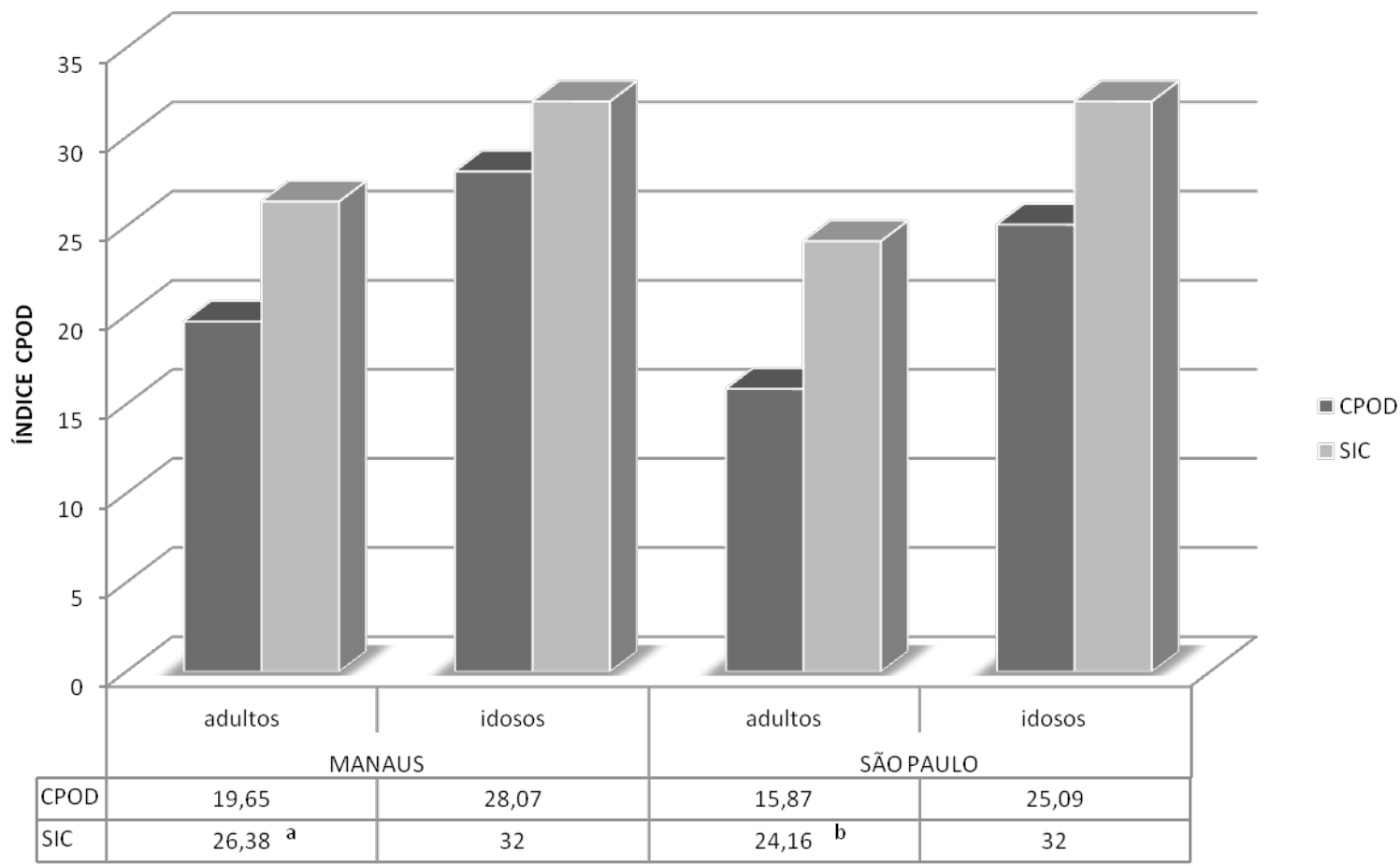

Gráfico 2 - Índice CPO-D e SiC em adultos e idosos de Manaus e São Paulo, 2010

$\mathrm{a}, \mathrm{b}$ Letras distintas na linha indicam diferença estatisticamente significante entre cada grupo etário. Fonte: Elaborado pelos autores, 2015.Elaborado pelos autores, 2015 
Quando os componentes do índice CPO-D foram desmembrados em cariados, perdidos e restaurados foi possível notar que o cariado foi o prevalente para todas as faixas etárias, com exceção do grupo de 15 a 19 anos em São Paulo, para o qual o componente restaurado foi o predominante. O Gráfico 3 demonstra a diferença entre os dois municípios relativa aos que tinham 5 e 12 anos de idade e aos que pertenciam à faixa etária de 15 a 19 anos. O componente perdido não apresentou diferença no caso de quem tinha 12 anos quando comparados os dois municípios. Também foi possível notar que o município de São Paulo apresentou maior porcentagem de elementos restaurados nos três grupos etários pesquisados ( $<<0,001)$.

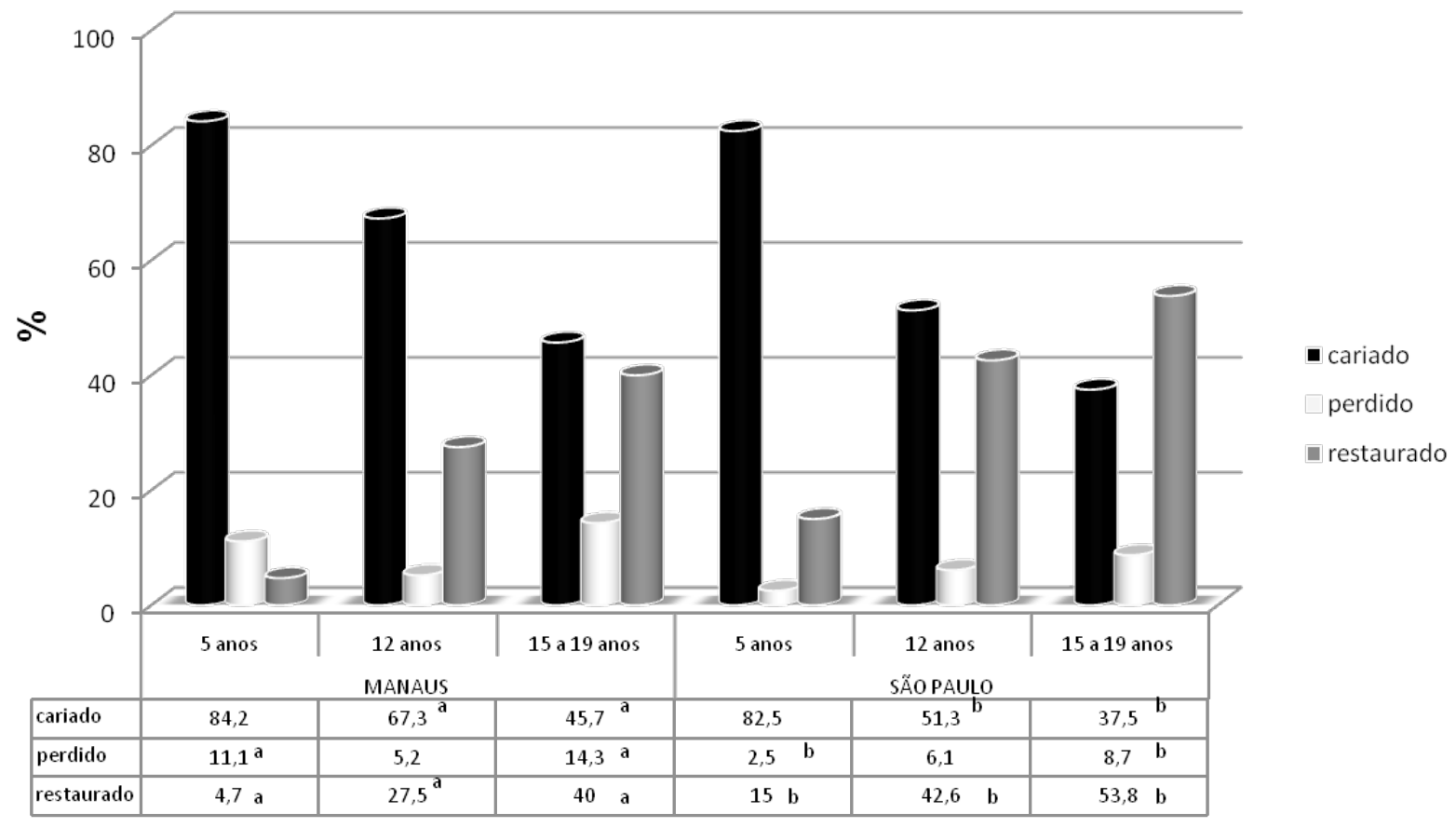

Gráfico 3 - Componentes do índice CPO-D aos 5, 12 e de 15 a 19 anos nos municípios de Manaus e São Paulo, 2010 a, bLetras distintas indicam diferença estatisticamente significante entre os grupos etários.

Fonte: Elaborado pelos autores, 2015.Elaborado pelos autores, 2015

Em relação à população adulta e idosa, em ambos os municípios o componente mais prevalente foi o de dentes perdidos, sendo que a maior porcentagem destes ocorreu no município de Manaus e a dos restaurados em São Paulo, como mostra o Gráfico 4. Não houve diferença entre as duas cidades no que diz respeito ao componente cariado. 


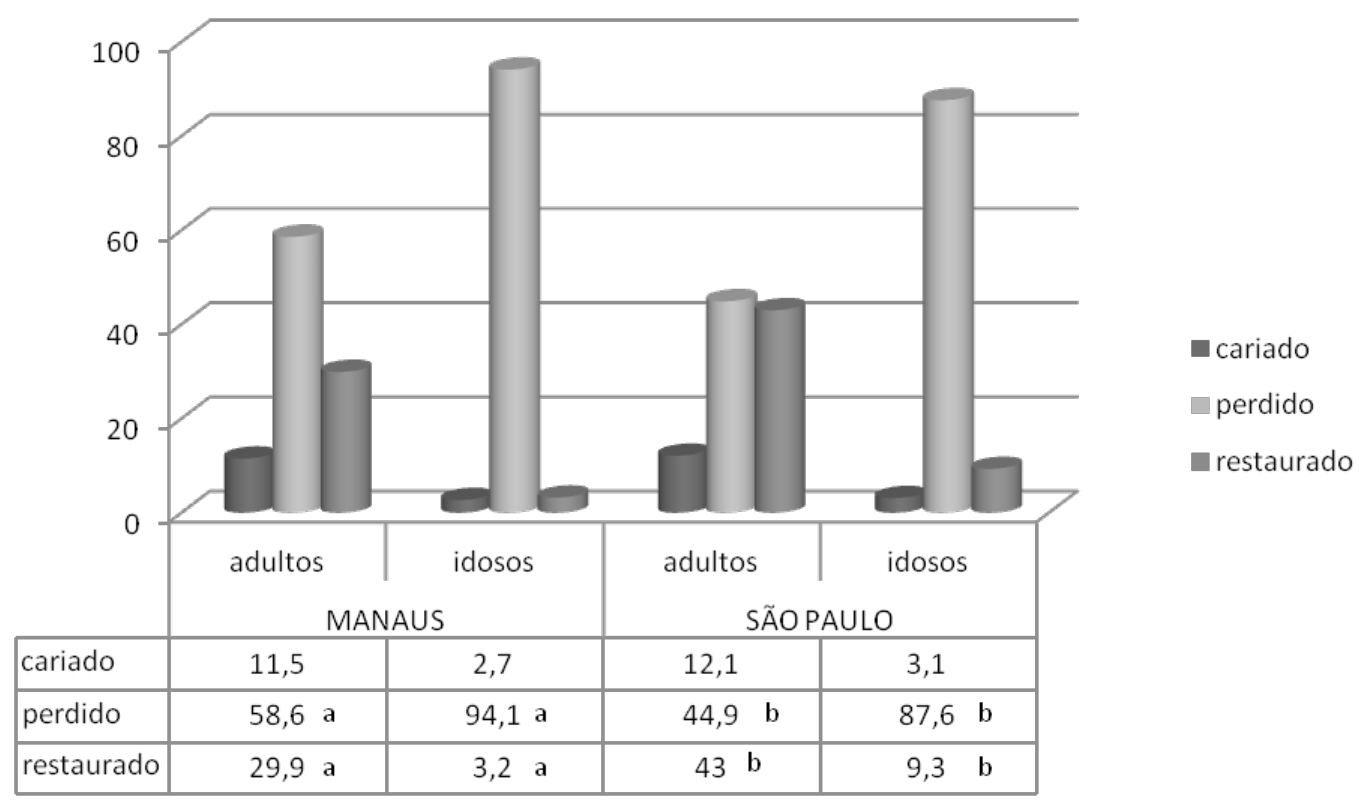

Gráfico 4 - Componentes do índice CPO-D de adultos e idosos nos municípios de Manaus e São Paulo, 2010 $a, b$ Letras distintas na linha indicam diferença estatisticamente significante entre os grupos etários.

Fonte: Elaborado pelos autores, 2015.

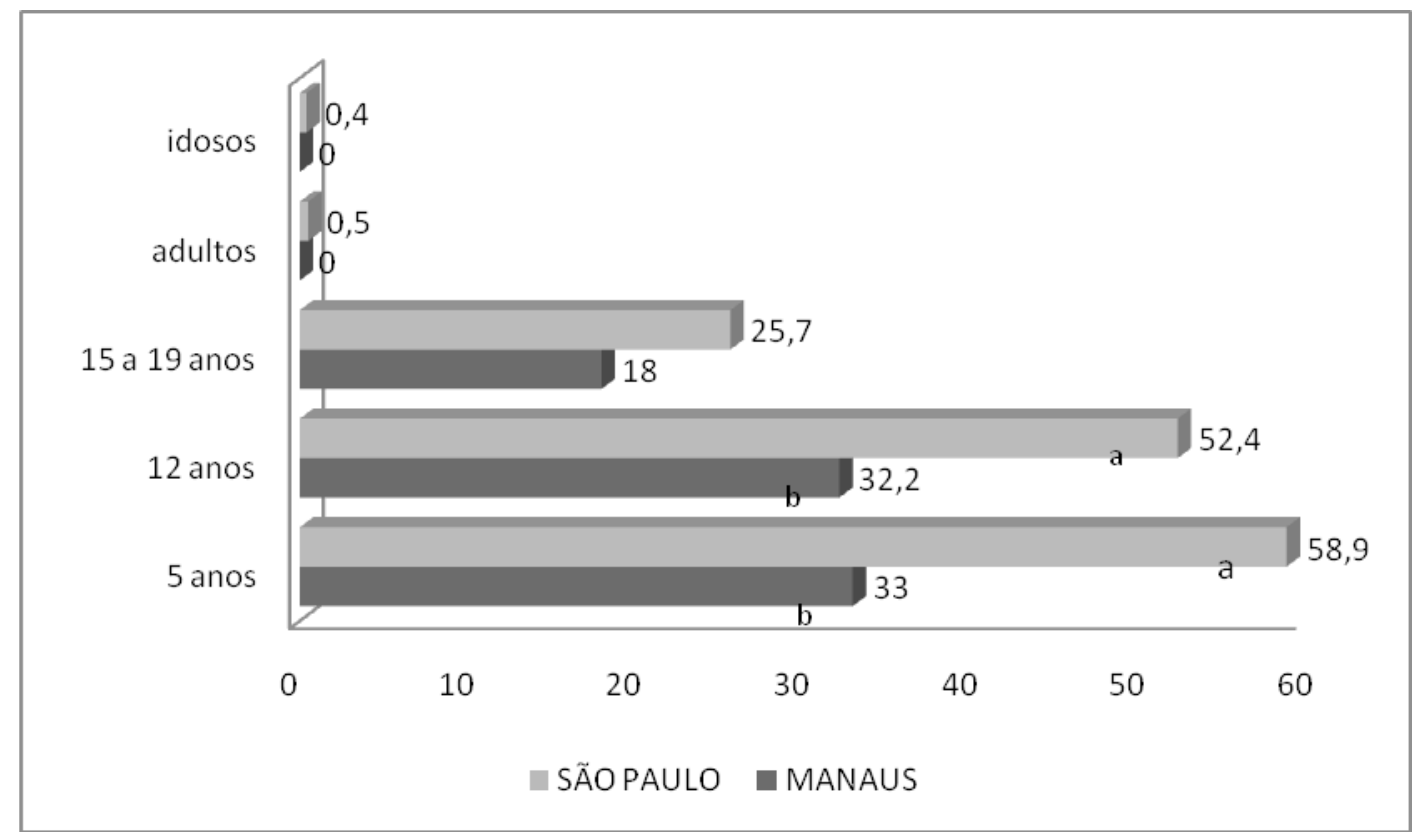

Gráfico 5 - Porcentagem de livres de cárie aos 5, 12, dos 15 aos 19 anos, adultos e idosos nos municípios de Manaus e São Paulo, 2010 $\mathrm{a}, \mathrm{b}$ Letras distintas indicam diferença estatisticamente significante entre os grupos etários.

Fonte: Elaborado pelos autores, 2015.Elaborado pelos autores, 2015

Os dados relativos aos livres de cárie demonstraram que o município de São Paulo possui maior porcentagem do que Manaus nos que têm 5 e 12 anos (p<0,001). Já na faixa etária de 15 a 19, e entre os adultos e idosos não houve diferença estatística significativa. 


\section{Discussão}

O quadro epidemiológico da cárie dentária no Brasil vem se modificando nas últimas décadas na maioria das faixas etárias estudadas, com exceção do grupo dos idosos, e a fluoretação da água de abastecimento público assim como os demais meios de utilização do flúor têm papel importante neste processo.

Como discutido em diversos artigos científicos, os efeitos preventivos do flúor são amplamente reconhecidos no controle da cárie dentária, e em ações de saúde pública são maiores quando a água é empregada como veículo ${ }^{11,15,16,17}$. Para Antunes ${ }^{18}$, "a fluoretação da água de abastecimento público é claramente uma estratégia de intervenção sobre os determinantes populacionais da cárie dentária, constituindo um dos elementos mais importantes da redução dos indicadores da doença no país e no exterior" e, segundo Ramires e Buzalaf ${ }^{19}$, em revisão de literatura, interromper a fluoretação da água acarreta aumento na prevalência de cárie de uma população de até $35 \%$ na dentição permanente.

Narvai e colaboradores ${ }^{15}$ citam que, nos Estados Unidos, na primeira década do século XXI houve uma redução global na prevalência e severidade da cárie, e que isso se deve, em grande parte, à exposição generalizada aos fluoretos, principalmente a partir da fluoretação da água potável. Informam ainda que, na Austrália, onde mais de 90\% da população recebem água de abastecimento público fluoretada, foi constatado que esta atividade continua a ser a medida mais eficaz e socialmente equitativa para prevenir cárie em todas as idades.

O nosso estudo demonstrou que o município de São Paulo tem menores índices de ceo-d e CPO-D quando se tem 5 e 12 anos de idade e maior porcentagem de crianças livres de cárie do que o município de Manaus, incluindo o grupo de polarização. Esses dados corroboram os dados de Cypriano e colaboradores ${ }^{16}$, para a região de Sorocaba, que encontraram diferença significante entre a média do índice ceod em crianças de 5 anos e de Tagliaferro e colaboradores ${ }^{10}$ para o índice CPOD, aos 12 anos em municípios de grande porte, com e sem água fluoretada.

No entanto, não houve diferença estatística no índice CPO-D e no grupo de polarização para a faixa etária de 15 a 19 anos entre os municípios pesquisados. Esses dados, somados aos do desmembramento dos componentes do índice CPO-D, demonstraram que mesmo sem diferença estatística na média do índice CPO-D, São Paulo apresentou melhores condições de saúde bucal. Nesse município houve porcentagem menor de dentes cariados e perdidos do que em Manaus. Estes resultados demonstram a dificuldade de se medir o impacto de medidas de controle da cárie dentária, que segundo Kumar $^{20}$ é caracterizada pela interação complexa de múltiplos fatores e o impacto imediato não é aparente, como observado nessa população beneficiada pela implementação da água de abastecimento fluoretada no município de São Paulo.

Além de ter a água fluoretada desde 1985, o município de São Paulo tem também um histórico de programas de atenção à saúde bucal, principalmente em escolas, o que pode ter contribuído com a diferença entre os componentes do índice CPO-D. O município de Manaus, por outro lado, não possui água fluoretada e não tem dados publicados sobre os programas de atenção à saúde bucal.

Dentro do histórico de programas de atenção à saúde bucal que o município de São Paulo possui, encontra-se o sistema incremental que foi disseminado por todo o estado de São Paulo a partir da década de 1950. Esse modelo foi utilizado até o início dos anos 1980 e pode explicar os resultados do estudo para a faixa etária de adultos de 35 a 44 anos de idade. Apesar de não demonstrar diferença estatística entre a experiência de cárie nos dois municípios, São Paulo apresentou porcentagem menor de dentes perdidos e maior de dentes restaurados. Os programas incrementais não conseguiram reduzir a incidência de cárie, uma vez que os métodos de controle da doença atingiam uma pequena parcela da população ${ }^{21}$. E, mesmo sendo caracterizados como misto (preventivo-curativo), enfatizavam a ação restauradora, assim, também não conseguiram diminuir o índice CPO-D, pois o componente cariado era substituído pelo obturado ${ }^{22}$. 
Ao analisar o SiC pode-se observar que, mesmo sem diferença estatística para o índice CPO-D entre os municípios, houve diferença no grupo de polarização entre os adultos. Esses resultados demonstraram que o grupo de alto risco foi beneficiado pela fluoretação, diminuindo a desigualdade em saúde bucal no município de São Paulo ${ }^{18}$.

O grupo dos idosos apresentou resultado semelhante ao dos adultos, tanto no que diz respeito às médias do índice CPO-D quanto à porcentagem de livres de cárie e dos outros componentes, com exceção do grupo de polarização, que não foi diferente entre os municípios. Em todos os aspectos o município de São Paulo demonstrou condição melhor de saúde bucal que Manaus, apesar da grande maioria ( $\mathrm{SP}=87,6 \%$ e MA= 94,1\%) ser de edêntulos, ou seja, pessoas que perderam seus dentes. Como descrito anteriormente, esse é o grupo que menos teve alterada a sua condição de saúde bucal ao longo dos anos no Brasil. A redução na experiência de cárie foi menos significativa, tendo em conta o caráter cumulativo das sequelas da doença. Os dados do SBBrasil 2010 para toda a população idosa brasileira demonstraram que o índice CPO-D praticamente não se alterou, ficando em 27,1 em 2010. Em 2003, a média era de 27,8, com a maioria correspondendo ao componente extraído ${ }^{14}$.

É prudente lembrar que o acesso ao dentifrício fluoretado, embora não substitua a fluoretação da água de abastecimento público, age como um fator coadjuvante na prevenção da doença cárie para qualquer faixa etária. No entanto, segundo Ramireze Buzalaf ${ }^{19}$, embora haja uma tendência a se afirmar que a ação da água fluoretada no controle da cárie esteja diminuindo, na verdade, o que está acontecendo é a ampliação de outros meios de utilização do flúor. E a fluoretação da água continua sendo efetiva para controlar a cárie dentária em populações de alto risco e sem acesso a outras fontes de flúor ${ }^{23}$.

\section{Conclusão}

Conclui-se que, segundo os dados do projeto SBBrasil 2010, o município de São Paulo apresentou melhores condições de saúde bucal do que Manaus e que a fluoretação das águas de abastecimento público tem papel importante nesse processo e deve ter continuidade.

\section{Referências}

1. World Health Organization. Guidelines for drinking-water quality: recommendations [Internet]. 3rd ed. Geneva; 2004 [cited 2016 Nov 18]. v.1.Disponível em: http://www.who.int/water sanitation health/ dwq/GDWQ2004web.pdf

2. CDC.Achievements in public health, 1900-1999: fluoridation of drinking water to prevent dental caries. MMWR. 1999;48(41):933-940.

3. Frazão P, Peres MA, Cury JA. Qualidade da água para consumo humano e concentração de fluoreto. Rev Saúde Pública. 2011; 45(4):964-73.

4. Brasil. Lei Federal no. 6.050, de 24 de maio de 1974. Dispõe sobre a obrigatoriedade da fluoretação das águas em sistemas de abastecimento. Diário Oficial da União 1974, 27 jul.

5. Brasil. Decreto no. 76.872. Regulamenta a Lei no. 6.050, de 24 de maio de 1974, que dispõe sobre a fluoretação da água em sistemas públicos de abastecimento. Diário Oficial da União 1975, 22 dez.

6. Truman BI, Gooch BF, Sulemana I, Gify HC,Horowitz AM, Evans CA, et al. Reviews of evidenceon interventions to prevent dental caries, oral and pharyngeal cancers, and sports-related craniofacial injuries. Am J PrevMed. 2002;23(1):21-54.

7. Australian Government. National Health and Medical Research Council. A systematic review of the efficacy and safety of fluoridation. Part A: review of methodology and results. Australian Government 2007.

8. Cury JA. Fluoretação da água: benefícios, riscos e sugestões. RevOdon Brasil Central. 1992;(2):32-33.

9. Narvai PC. Fluoretação da água: heterocontrole no Município de São Paulo no período 1990-1999.

Revista Brasileira de Odontologia em Saúde Coletiva. 2000;(2):50-56. 
10. Tagliaferro EP, Cypriano S, Sousa MLR, Wada WS. Caries experience among school children in relation to community fluoridation status and town size. Acta Odontol Scand. 2004 jun;62(3):124-8.

11. Rihs LB, Sousa MLR, Cypriano S. Cárie dentária em adultos em locais com e sem água fluoretada na região de Campinas, São Paulo. Rev. Fac. Odontol. Porto Alegre. 2007;48(1/3):69-79.

12. Brasil. IBGE. Instituto brasileiro de geografia e estatística. Censo 2010 [citado 2 mar. 2016]. Disponível em: http://www.ibge.gov.br/home/estatistica/populacao/censo2010/default.shtm

13. Narvai PC. Vigilância sanitária da fluoretação das águas de abastecimento público no município de São Paulo, Brasil, no período de 1990-1999. (tese livre docência). São Paulo: Faculdade de Saúde Pública da Universidade de São Paulo; 2001. Disponível em: http://www.teses.usp.br/teses/disponiveis/ livredocencia/6/tde-21092007-114848/pt-br.php

14. Brasil. Ministério da Saúde. SBBrasil 2010: Pesquisa Nacional de Saúde Bucal. Resultados principais. 2012. Disponível em http://bvsms.saude.gov.br/bvs/publicacoes/pesquisa nacional saude bucal.pdf

15. Narvai PC, Frias AC, Fratucci MVB, Antunes JLF, Carnut L, Frazão P.Fluoretação da água em capitais brasileiras no início do século XXI: a efetividade em questão. Saúde Debate. 2014 jul set; 38(102):562-71.

16. Cypriano S, Pecharki DP, Sousa MLR, Wada RS. A saúde bucal de escolares residentes em locais com ou sem fluoretação nas águas de abastecimento público na região de Sorocaba, São Paulo, Brasil. Cad. Saúde Pública.2003 jul-ago;19(4):1063-1071.

17. Meirelles MPMR, Sousa MLR. A importância da fluoretação das águas de abastecimento em municípios de pequeno porte na região sudeste do estado de São Paulo. Rev Fac Odonto Porto Alegre. 2005;46(2):15-19.

18. Antunes JLF, Narvai PC. Políticas de saúde bucal no Brasil e seu impacto sobre as desigualdades em saúde.Rev Saúde Pública. 2010;44(2):360-5.

19. Ramirez I, Buzalaf MAR. A fluoretação da água de abastecimento público e seus benefícios no controle da cárie dentária - cinqüenta anos no Brasil. Ciência e Saúde Coletiva. 2007;12(4):1057-65.

20. Kumar JV.Is water fluoridation still necessary?.Adv Dent Res. 2008 Jul;20:8-12.

21. Pinto VG. Revisão sobre o uso e segurança do flúor. Rev Gaucha Odontol. 1993;41(5):263-6.

22. Nickel DA, Lima FG, Silva BB. Modelos assistenciais em saúde bucal no Brasil. Cad. Saúde Pública.2008 Fev;24(2):241-246.

23. Horowitz HC. The future of the water fluoridation and other systemic fluorides. J Dental Res. 1990; 69:760-764. 